\title{
Novel HBsAg markers tightly correlate with occult HBV infection and strongly affect HBsAg detection
}

\author{
Valentina Svicher ${ }^{\mathrm{a}, 1}$, Valeria Cento ${ }^{\mathrm{a}, 1}$, Martina Bernassola ${ }^{\mathrm{b}}$, Maria Neumann-Fraune ${ }^{\mathrm{c}}$, \\ Formijn Van Hemert ${ }^{\mathrm{d}}$, Mengjie Chen ${ }^{\mathrm{e}}$, Romina Salpini ${ }^{\mathrm{a}}$, Chang Liu ${ }^{\mathrm{e}}$, Roberta Longo ${ }^{\mathrm{b}}$, Michela Visca ${ }^{\mathrm{b}}$, \\ Sara Romano ${ }^{\mathrm{b}}$, Valeria Micheli ${ }^{\mathrm{f}}$, Ada Bertoli ${ }^{\mathrm{g}}$, Caterina Gori ${ }^{\mathrm{h}}$, Francesca Ceccherini-Silberstein ${ }^{\mathrm{a}}$, \\ Cesare Sarrecchia ${ }^{g}$, Massimo Andreoni ${ }^{g}$, Mario Angelico ${ }^{g}$, Antonella Ursitti ${ }^{\mathrm{b}}$, Alberto Spanò ${ }^{\mathrm{b}}$, \\ Jing Maria Zhang ${ }^{\mathrm{e}}$, Jens Verheyen ${ }^{\mathrm{c}}$, Giuseppina Cappiello ${ }^{\mathrm{b}}$, Carlo Federico Perno ${ }^{\mathrm{a}, \mathrm{g}, *}$ \\ a Department of Experimental Medicine and Biochemical Sciences, University of Rome "Tor Vergata" Rome, Italy \\ ${ }^{\mathrm{b}}$ Department of Microbiology, "S. Pertini" Hospital, Rome, Italy \\ ${ }^{\mathrm{c}}$ Institute of Virology, University of Cologne, Cologne, Germany \\ d Center for Infection and Immunity Amsterdam (CINIMA), Academic Medical Center, University of Amsterdam, Amsterdam, The Netherlands \\ e Department of Statistics, Yale University, USA \\ ${ }^{\mathrm{f}}$ Microbiology and Virology, "L. Sacco" Hospital, Milan, Italy \\ ${ }^{g}$ University Hospital of Rome "Tor Vergata", Rome, Italy \\ h Antiretroviral Therapy Monitoring Unit, "L. Spallanzani" Hospital, Rome, Italy
}

\section{A R T I C L E I N F O}

\section{Article history:}

Received 18 August 2011

Revised 26 October 2011

Accepted 30 October 2011

Available online 9 November 2011

These data have been presented in part at the 46th Annual Meeting of the European Association for the Study of the Liver (EASL) held in Berlin, Germany, March 30-April 3, 2011 where the abstract was scored among the $10 \%$, and at the 2nd International HIV and Viral Hepatitis Drug Resistance

Workshop held in Los Cabos, Mexico, June 8-11, 2011.

\section{Keywords:}

HBV

Occult infection

HBsAg structure

HBsAg detection

\begin{abstract}
A B S T R A C T
Occult HBV infection (OBI) is a threat for the safety of blood-supply, and has been associated with the onset of HBV-related hepatocellular carcinoma and lymphomagenesis. Nevertheless, genetic markers in HBsAg (particularly in D-genotype, the most common in Europe) significantly associated with OBI in vivo are missing. Thus, the goal of this study is to define: (i) prevalence and clinical profile of OBI among blood-donors; (ii) HBsAg-mutations associated with OBI; (iii) their impact on HBsAg-detection. OBI was searched among 422,278 blood-donors screened by Nucleic-Acid-Testing. Following Taormina-OBI-definition, 26 (0.006\%) OBI-patients were identified. Despite viremia $<50 \mathrm{IU} / \mathrm{ml}$, HBsAg-sequences were obtained for $25 / 26$ patients (24/25 genotype-D). OBI-associated mutations were identified by comparing OBI-HBsAg with that of 82 chronically-infected (genotype-D) patients as control. Twenty HBsAg-mutations significantly correlated for the first time with OBI. By structural analysis, they localized in the major HBV B-cell-epitope, and in HBsAg-capsid interaction region. 14/24 OBI-patients (58.8\%) carried in median 3 such mutations (IQR:2.0-6.0) against 0 in chronically-infected patients. By co-variation analysis, correlations were observed for R122P $+\mathrm{S} 167 \mathrm{~L}(\mathrm{phi}=0.68, P=0.01), \mathrm{T} 116 \mathrm{~N}+\mathrm{S} 143 \mathrm{~L}$ ( $\mathrm{phi}=0.53$, $P=0.03$ ), and $\mathrm{Y} 100 \mathrm{~S}+\mathrm{S} 143 \mathrm{~L}(\mathrm{phi}=0.67, p<0.001)$.

Mutants (obtained by site-directed mutagenesis) carrying T116N,T116N + S143L, R122P, R122P + Q101R, or R122P + S167L strongly decreased HBsAg-reactivity $(54.9 \pm 22.6 \mathrm{~S} / \mathrm{CO}, 31.2 \pm 12.0 \mathrm{~S} / \mathrm{CO}, 6.1 \pm 2.4 \mathrm{~S} / \mathrm{CO}$, $3.0 \pm 1.0 \mathrm{~S} / \mathrm{CO}$ and $3.9 \pm 1.3 \mathrm{~S} / \mathrm{CO}$, respectively) compared to wild-type (306.8 $\pm 64.1 \mathrm{~S} / \mathrm{CO})$. Even more, Y100S and Y100S + S143L supernatants show no detectable-HBsAg (experiments in quadruplicate).

In conclusions, unique HBsAg-mutations in genotype-D, different than those described in genotypes $\mathrm{B} / \mathrm{C}$ (rarely found in western countries), tightly correlate with OBI, and strongly affect HBsAg-detection. By altering HBV-antigenicity and/or viral-particle maturation, they may affect full-reliability of universal diagnostic-assays for HBsAg-detection.
\end{abstract}

(c) 2011 Elsevier B.V. All rights reserved.

\section{Introduction}

Chronic overt hepatitis B is characterized by detectable hepatitis B surface antigen (HBsAg) in the serum, while occult HBV infection

\footnotetext{
* Corresponding author. Address: Department of Experimental Medicine and Biochemical Sciences, University of Rome "Tor Vergata", Via Montpellier 1, 00133 Rome, Italy. Tel.: +3906 72596551; fax: +3906 72596039.

E-mail address: cf.perno@uniroma2.it (C.F. Perno).

1 Equally contributed to the manuscript.
}

(OBI) is defined by the persistence of HBV-DNA in the liver tissue (and in some cases also in the serum) in absence of HBsAg (Raimondo et al., 2010). OBI is one of the most challenging topics in the field of viral hepatitis. Indeed, OBI can be transmitted in cases of transfusion of blood products, as well as during orthotopic liver transplantation, and it has been associated with the progression of chronic liver damage towards cirrhosis and hepatocellular carcinoma despite the apparently low HBV-replication rate (Raimondo 
et al., 2008). Indeed, OBI has been shown to maintain the pro-oncogenic properties typical of the chronic infection, and has been identified in up to $70.4 \%$ of HBsAg-negative patients with hepatocellular carcinoma (Fang et al., 2009). In addition, it has been shown that lymphotropism is a major feature of OBI, and that a relationship may exist between OBI and lymphomagenesis (Engels et al., 2010). Finally, patients with OBI are at potential risk of HBV reactivation during immune-suppressive therapy in the future, especially in regimens containing rituximab (Hoofnagle, 2009). This supports the need to set up effort for a correct detection of OBI.

OBI is also a potential threat for the safety of blood supply. The prevalence of OBI in blood donors has been reported in some European Countries such as Poland, Germany, and France (Brojer et al., 2006; Katsoulidou et al., 2009; Levast et al., 2010), while in others, such Italy, recent data are limited (Velati et al., 2008; Dettori et al., 2009). This information is critical in order to set up optimized transfusion services.

Several host and viral factors have been hypothesized for the OBI pathogenesis (Blum et al., 1991; Chaudhuri et al., 2004; Zaaijer et al., 2008; Vivekanandan et al., 2008; El Chaar et al., 2005). Nevertheless, conclusive studies on HBV genetic markers (in particular in the $\mathrm{HBsAg}$ ) responsible for $\mathrm{OBI}$ in vivo are lacking. This can be due to a number of factors: (1) The number and type of patients studied: the majority of reports included a limited number of patients infected with different HBV genotypes, thus making difficult to extrapolate the effect of mutations from the different genetic background; (2) The technology used for viral genome sequencing: not widely available for low level viremia; (3) The analysis of different fragments of HBV genome and the methodologies used to characterize HBV genetic variability: the majority of studies reported single mutations correlated with occult HBV infection without providing a more complex level of information regarding the cluster of mutations underlying occult HBV infection in vivo.

Thus, and despite its clinical relevance, the genetic characteristics in HBsAg from patients with occult HBV is still poorly known. This is particularly true for HBV, D-genotype infection that is the most prevalent in Mediterranean Countries including Southern Europe.

In this light, using extensive sequence analyses, unique computational methods, and phenotypic characterization, this study is aimed (i) at defining the extent of occult HBV infection in asymptomatic blood donors in a Country with low HBV endemicity, (ii) at identifying HBsAg-genetic markers specifying occult HBV infection in vivo in one of the largest homogenous cohort of OBI-patients so far analyzed, (iii) as proof of concept, at investigating the impact of such markers on the detection of HBsAg in the supernatants of cell culture experiments.

Overall, this information can provide new insights regarding the pathogenesis of OBI, and can be used to further improve the rate of true-positive results by standard assay for HBsAg detection in diagnostic practice.

\section{Methods}

\subsection{Patients}

The present study was carried out in two cohorts involving a total of 422,278 regular and occasional blood donors, screened for HCV, HIV-1/2 and HBV infection by Nucleic Acid Testing (NAT) at a reference center for transfusion security in Lazio region. The first cohort involved blood donors who donated blood between December 2004 and April 2007 These samples were tested using polymerase chain reaction (PCR)-based $\mathrm{HIV}-1 / \mathrm{HCV} / \mathrm{HBV}$ test (COBAS Ampliscreen, Roche Diagnostics, Branchburg, NJ; lower limit of detection [LOD]: $5 \mathrm{IU} / \mathrm{mL}, 95 \%$ confidence interval: $3-13 \mathrm{IU} / \mathrm{mL}$ ) in pools of 20 samples, generated with Tecan Genesis pipetting machine (Tecan Schweiz AG, Männedorf, Switzerland). HBV-DNA reactive pools were resolved by minipools of 5 samples testing. Donations of minipool, found to be NAT-reactive, were tested individually to identify the positive unit.

The second cohort of donors, who donated blood between March 2007 and December 2008, were screened in minipools of 6 samples using the COBAS Taqscreen MPX (Roche Molecular Systems, Branchburg, NJ; LOD: $3.1 \mathrm{IU} / \mathrm{mL}, 95 \%$ confidence interval: 1.7-7.8 IU/mL) test on the COBAS s201 system for the direct detection of HIV1-2/HCV/HBV (Roche Instrument Center, Rotkreuz, Switzerland). When a minipool was reported as reactive, the 6 donation were individually tested with the COBAS Taqscreen MPX test on the COBAS s201 system as a part of the resolution process to identify the reactive and non-reactive donor sample.

Any individual specimens found to be reactive was tested using HIV1/HCV/HBV COBAS Ampliscreen (Roche Diagnostics, Branchburg, NJ) for viral discrimination. HBV-DNA was quantified by quantitative PCR COBAS Taqman High Pure System HBV (Roche Molecular Systems, Branchburg, NJ; LOD: $6 \mathrm{IU} / \mathrm{mL}$ ) between December 2004 and December 2005 and by COBAS Ampliprep/COBAS Taqman HBV test (Roche Molecular Systems, Branchburg, NJ; LOD: $12 \mathrm{IU} / \mathrm{mL}$ ) between November 2005 and December 2008.

Confirmed HBV-DNA positive samples were retested for serological markers of HBV infection (HBsAg, anti-HBc, anti-HBc-IgM, $\mathrm{HBeAg}$, anti-HBe, anti-HBs) using specific tests on the Roche Elecsys 2010 immunoassay analyzer (Roche Diagnostics, Branchburg, NJ).

\section{2. $H B s A g-D N A$ sequencing}

HBsAg-DNA sequencing was performed following the methodology reported in (Weinberger et al., 1999). Specific primers and amplification protocols are reported in detail in Supplementary Text 1. Translated HBsAg-sequences were generated and aligned using Bioedit 7.0 software and CLUSTALW1.8. All sequences are in process to be submitted to GenBank. A phylogenetic analysis was performed to exclude possible contaminations.

\subsection{Genetic variability analysis}

Shannon Entropy $\left(\mathrm{Sn}=-\Sigma_{i}\left(p_{i} \ln p_{i}\right) / \ln N\right)$ was calculated, where $p_{i}$ was the frequency of each distinct nucleotide sequence and $N$ was the total number of sequences analyzed. Statistical significance was assessed trough Wilcoxon rank test. Evolutionary divergence of HBsAg-sequences was estimated as the extent of nucleotide substitutions per site determined by the HKY model of MEGAv5.0 (Tamura et al., 2011), applying a gamma distribution with shape parameter $=0.8$. The nested codon models 7 and 8 of PAML 3.15 (Yang, 2007) were used to calculate $\mathrm{dN} / \mathrm{dS}$ ratio values. The nwk user tree was generated in MEGA5 (amino acid alignment, JTT replacement model), CodonFreq F3x4 was chosen and gaps were treated as "missing data" (cleandata $=0$ ). Likelihood Ratio Tests were performed to verify a better fit of the data with model 8 (extra size class $(\mathrm{dN} / \mathrm{dS}>1$ ) with respect to the null-hypothesis (model 7, 10 size classes, $\mathrm{dN} / \mathrm{dS} \leqslant 1$ ).

\subsection{Statistical analysis}

\subsubsection{Mutation prevalence}

To assess the association of HBsAg-mutations with occult infection, we calculated the prevalence of all amino acids (including the wild-type amino acids) found at each HBsAg-position in the 24 sequences from occult infected patients, and 82 sequences from chronically infected patients. We then performed chi-squared tests of independence with a Benjamini-Hochberg correction (false discovery rate, $F D R=0.05$ ) to identify statistically significant differences in frequency between the two groups of patients. 


\subsubsection{Mutation covariation}

Binomial-correlation coefficient (phi) was calculated to assess covariation among mutations associated with occult infection (Svicher et al., 2009). Statistically significant pairs of mutations were identified by Fisher's exact test, corrected for multiple-testing by Benjamini-Hochberg method (FDR $=0.05)$.

\subsubsection{Cluster analysis}

In order to identify and summarize higher-order interactions of mutations, we transformed the pairwise phi correlation coefficients into dissimilarity values. A dendrogram was then computed by hierarchical clustering, and its stability was assessed from 100 bootstrap replicates. The details of this explorative data analysis procedure have been described elsewhere (Svicher et al., 2009).

\subsection{Bayesian variable partition model and recursive model selection}

Bayesian Variable Partition Model and Recursive Model Selection were carried out according to the methodology described in Zhang et al. (2010). A detailed description of these models is reported in Supplementary Text 2.

\subsection{HBsAg three-dimensional structure prediction}

An in silico generated tertiary structure of HBV S-protein was available including the amino acid residues comprising the interface composition of a surface-capsid complex (Roy et al., 2010). The amino acid replacements described to be associated with occult HBV infection (this paper) were manually introduced into the sequence. To generate a 3D model of surface protein carrying these mutations, the file was submitted to I_TASSER for homology modeling with the original "D-consensus" structure as a custom modeling template. Residues are colored according to membrane topology, A-determinant size and surface-capsid interface composition as described in Roy et al. (2010). Hydrophobicity profiles were constructed using values normalized between 0 and 1 (Black and Mould, 1991). A sliding window of 5 residues with a step size of 1 was applied.

\subsection{Site-specific mutagenesis and HBsAg detection assay}

The expression vector pCHsAg (genotype D) under the control of cytomegalovirus immediate early promoter-enhancer was used as wild type control and as backbone vector for the expression of $\mathrm{HBsAg}$. Single or double HBsAg-mutations were introduced by site directed mutagenesis in the expression vector and confirmed by sequence reactions. Wild type and $\mathrm{HBsAg}$ mutants were tested by transfection of human $\mathrm{HuH7}$ cells with pCHsAg plasmids using FuGene6 (Roche diagnostics GmbH, Mannheim, Germany). The supernatant was collected after $72 \mathrm{~h}$ and tested using the ABBOTT Architect HBsAg (Qual) assay. HBsAg was tested in cell-supernatant obtained from four independent experiments for each mutant and wild type. These results were used to calculate the mean value and standard deviation for each HBsAg mutant.

\section{Results}

\subsection{Prevalence of occult infection and description of the study population}

The frequency of HBV-DNA positive/HBsAg negative donations was assessed in a large cohort of blood donors recruited in an important reference center for transfusion security in Central Italy.

Among 422,278 blood donors tested, 269 (0.06\%) were positive for serum HBV-DNA by NAT procedure. After HBsAg testing, 243/
Table 1

Demographic and serological characteristics of the study population.

\begin{tabular}{ll}
\hline Characteristic & Patients with occult HBV infection \\
\hline Patients, $N$ & 26 \\
Age (years), median (IQR) & $56.0(51.1-62.6)$ \\
Male, $N(\%)$ & $22(84.6)$ \\
Italian nationality, $N(\%)$ & $25(96.2)$ \\
HBV genotype & \\
D & $24(92.3)$ \\
A & $1(3.8)$ \\
Undetermined & $1(3.8)$ \\
Type of blood donation & \\
First time donors & $10(38.5)$ \\
Habitual donors & $16(61.5)$ \\
$N(\%)$ of patients with serum HBV-DNA & \\
$\leqslant 2$ UI/ml & $22(84.6)$ \\
$>2$ UI/ml & $4(15.4)^{\mathrm{b}}$ \\
ALT (IU/ml), median (IQR) & $21.5(19.0-29.3)$ \\
Serological characteristics of patients & \\
HBcAb IgG,$N(\%)$ & $26(100)$ \\
HBcAb IgM ${ }^{+}, N(\%)$ & 0 \\
HBeAg ${ }^{+}, N(\%)$ & 0 \\
HBeAb ${ }^{+}, N(\%)$ & $19(73.1)$ \\
HBsAg (S/CO), median, (IQR) & $0.46(0.38-0.59)$ \\
HBsAb ${ }^{+}, N(\%)$ & $11(42.3)$ \\
HBsAb (mIU/ml), median, (IQR) & $28.0(20.0-54.0)$ \\
\hline
\end{tabular}

$\mathrm{IQR}$, interquartile range.

a Serum HBV-DNA was determined by COBAS TaqMan High Pure System and by COBAS AmpliPrep/TaqMan HBV.

b Viremia ranges from 30 to $50 \mathrm{IU} / \mathrm{ml}$.

269 (90.3\%) patients were HBV-DNA-positive and HBsAg-positive (thus with diagnosis of overt chronic HBV hepatitis), while the other 26 patients $(0.006 \%)$ were HBV-DNA-positive and HBsAgnegative. No significant difference in the prevalence of HBVDNA-positive and HBsAg-negative patients was observed in the two cohorts of blood donors $(0.005 \%$ in the first cohort screened between 2004 and 2007 versus $0.008 \%$ in the second cohort screened between 2007 and 2008). Following the position paper issued by the recent meeting of experts in Taormina (Raimondo et al., 2008), OBI is associated with undetectable or low level HBV-DNA $(<200 \mathrm{IU} / \mathrm{ml})$ in the serum of HBsAg negative individuals. For $22 / 26$ patients (84.6\%), serum HBV-DNA quantitation was $<12 \mathrm{IU} / \mathrm{ml}$, while for the remaining 4 patients, viremia ranged from 30 to $50 \mathrm{IU} / \mathrm{ml}$ (Table 1 ). ALT values were in the normal range. Therefore, these $26 \mathrm{HBV}$-DNA-positive and HBsAg-negative patients were considered as occult HBV-infected patients.

Demographic and serological characteristics of the 26 blood donors with OBI are summarized in Table 1 . The majority were Italian (25/26, 96.2\%), and male (22/26, 84.6\%). All blood donors with OBI were anti-HBc positive, and anti-HBc IgM negative, thus indicating that they were not in the window phase of acute hepatitis B infection. Eleven out of 26 (42.3\%) patients also showed low level of protective anti-HBs (>10 m UI/ml) (Median [IQR] = 28.0 [20.054.0]).

Despite HBV-DNA < $50 \mathrm{IU} / \mathrm{ml}$, HBsAg-DNA sequences were obtained for 25/26 (96\%). Direct HBsAg sequencing showed that genotype-D was dominant $(24 / 25,96.0 \%)$, while genotype-A was present only in 1 patient.

\section{2. $H B s A g$ genetic markers underlying $O B I$ in vivo}

HBsAg genetic characteristics underlying $\mathrm{OBI}$ in vivo were investigated. In order to make data more clear and readable, the analysis was focused on 24 patients with occult HBV D-genotype infection (thus excluding the only genotype-A patient). A group of 82 randomly-selected, chronically HBV D-genotype infected 

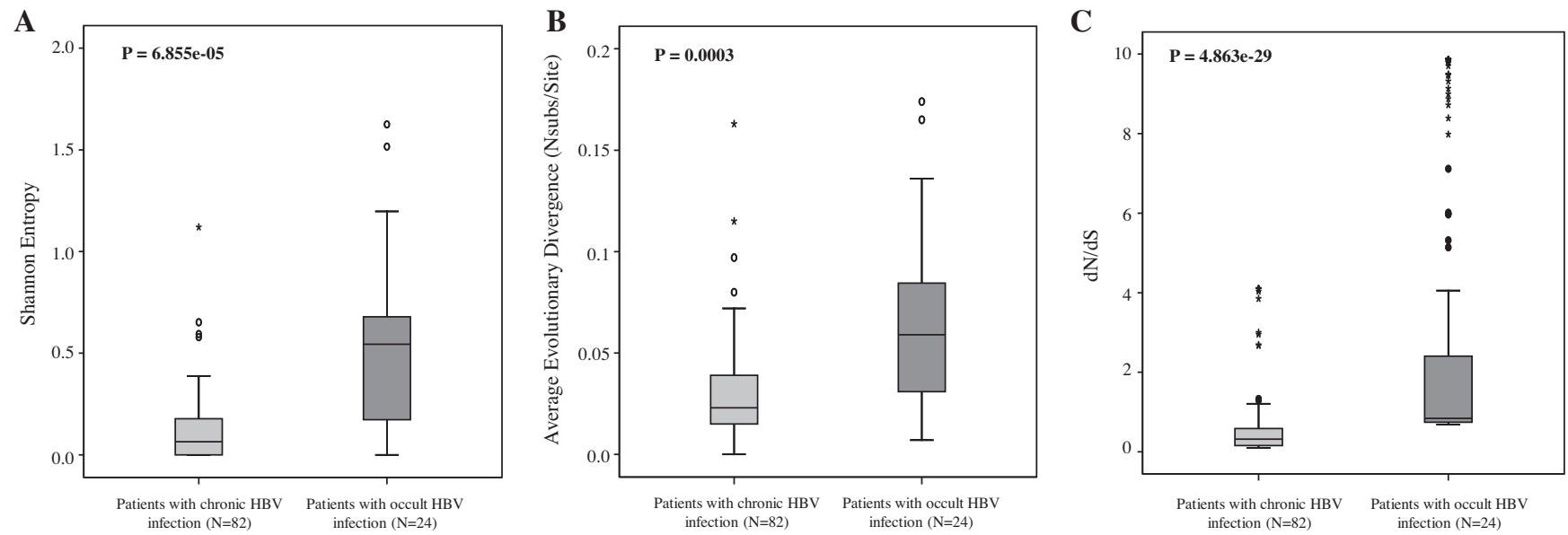

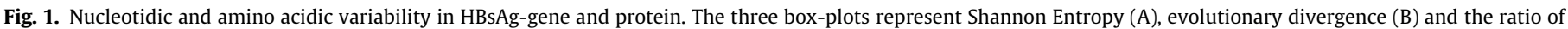
non-synonymous (dN) and synonymous (dS) substitutions rates (C) among patients with chronic infection and OBI. P-values were calculated by Mann-Whitney Test.

patients was used as control. All chronically infected patients were HBsAg-positive, anti-HBc positive with a median (IQR) serum HBVDNA of 5.9 (3.3-6.2) $\log \mathrm{IU} / \mathrm{ml}$ (Supplementary Table S1). All of them were naïve to any anti-HBV drug-treatment.

A higher degree of genetic variability was found in HBsAg-sequences from OBI compared to patients with chronic HBV infection. Indeed, both the median Shannon Entropy, the median evolutionary rate, and the $\mathrm{dN} / \mathrm{dS}$ ratio were remarkably higher in patients with OBI than in patients with chronic HBV infection (median Shannon Entropy (IQR): $0.29(0.00-0.54)$ versus 0.07 (0.00-0.18), $P=0.000068$; median evolutionary divergence (IQR): $0.06(0.03-0.08)$ versus $0.02(0.02-0.04), P=0.0003 ; \mathrm{dN} / \mathrm{dS}: 0.84$ $(0.74-2.36)$ versus $\left.0.32(0.15-0.59), P=4.863 \cdot 10^{-29}\right)$ (Fig. 1 ).

This result prompted us to investigate the existence of HBsAg markers specifying OBI in vivo.

Overall, 20 HBsAg-mutations were found for the first time significantly correlated with occult HBV D-genotype infection in vivo (Fig. $2 \mathrm{~A}$ ) ( $P$-values from $10^{-2}$ to $10^{-4}$ after correction for multiple comparison). Fourteen out of 24 (58.8\%) patients with OBI (vs. only $1 / 82$ chronic infected patients) carried $\geqslant 1$ of these mutations (median number [IQR]: 3.0 [2.0-6.0]). Conversely, in our cohort of 82 chronically-infected patients, such mutations were completely absent (0/82 for Y100S, P105R, T116N, P120L, R122P, T126I, Y134C, S143L, S167L, R169H) or nearly absent (1/ 82 for T115N, P127H, S174N, V177A, 2/82 for Q129P, and 3/82 for Q101R, P127L, L175S, one mutation per patient). Their prevalence ranged from $8.3 \%$ to $20.8 \%$ in OBI patients, compared to $0-3.7 \%$ in the 82 chronically-infected patients.

A Bayesian variable partition model (Zhang et al., 2010) was then applied to further confirm whether HBsAg-positions are associated with OBI. All HBsAg-mutations identified in the above-mentioned analysis were fully confirmed by this model. Indeed, all mutations showed a posterior probability ranging from 0.75 to 1 , indicating a strong association with occult infection (data not shown).

\subsection{Localization of HBsAg residues in the predicted HBsAg three- dimensional structure}

To gain insight into effects of OBI-associated amino acid replacements on $\mathrm{HBsAg}$, we introduced these replacements in the sequence and built a 3D-model of mutant HBsAg for comparison with D-consensus HBsAg. Fig. 2B shows that OBI-associated residues are mainly located at positions involved in antibody recognition and capsid interaction. In the 3D-structure of mutant HBsAg (Fig. 2C), the luminal loop including the A-determinant region dis- plays a "collapse" towards the hydrophobic center of the molecule. The architecture at the capsid-interaction regions is less modified. A similar view is presented by the hydrophobicity profiles (Fig. 2D) showing a replacement of hydrophilic into more hydrophobic residues particularly at the A-determinant region (110-140).

\subsection{Specific associations among HBsAg markers}

A further step of this study was to investigate the complex interaction patterns of $\mathrm{HBsAg}$-mutations associated with $\mathrm{OBI}$ in vivo. This is the first study addressing this point.

\subsubsection{Associations among $\mathrm{HBs} A g$-mutations}

To identify significant patterns of pairwise correlations between mutations at the above mentioned positions, we calculated the binomial correlation coefficient (phi) and its statistical significance for each pair of mutations. A positive and statistically significant correlation between mutations at two specific positions $(0<$ phi $<1, p<0.05)$ indicates that these two positions co-evolve; thus it indicates that the co-occurrence of mutations is not due by chance. A strong and significant positive association of S143L with either T116N (phi $=0.68$ ) or Y100S (phi $=0.66$ ), and of R122P with either T127S (phi=0.84), Q101R (phi = 0.56), or S167L ( $\mathrm{phi}=0.56$ ) was found. A significant correlation was also observed between L175S and V177A, both localized in the HBsAg hydrophobic C-terminus ( $\mathrm{phi}=0.56$ ).

\subsubsection{Clusters of correlated mutations}

Because pairwise analysis suggested that some novel mutations are associated with specific evolutionary pathways, an average linkage hierarchical agglomerative cluster analysis was performed to investigate this hypothesis in more detail.

The topology of the dendrogram (Supplementary Fig. S1) highlighted the existence of two distinct clusters of HBsAg-mutations in OBI. The first cluster included the mutations T116N and S143L (bootstrap value $=0.51$ ), that were linked to Y100S (bootstrap $=0.54$ ). The second pattern included the mutations $S 167 \mathrm{~L}$, and Q101R that were linked to T127S and R122P (bootstrap value $=0.50$ ).

\subsection{Phenotypic analysis of HBsAg-mutation's patterns in occult infection}

Cluster analysis highlighted the existence of specific cluster underlying OBI in vivo. As proof of concept, the ability of these 
A

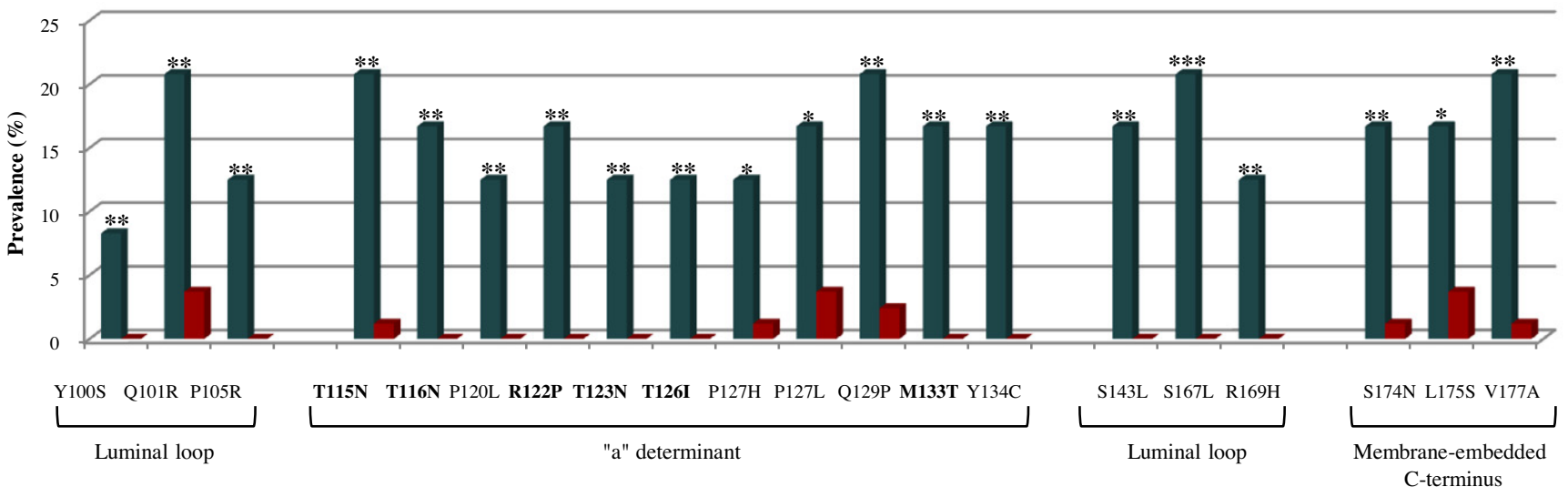

B

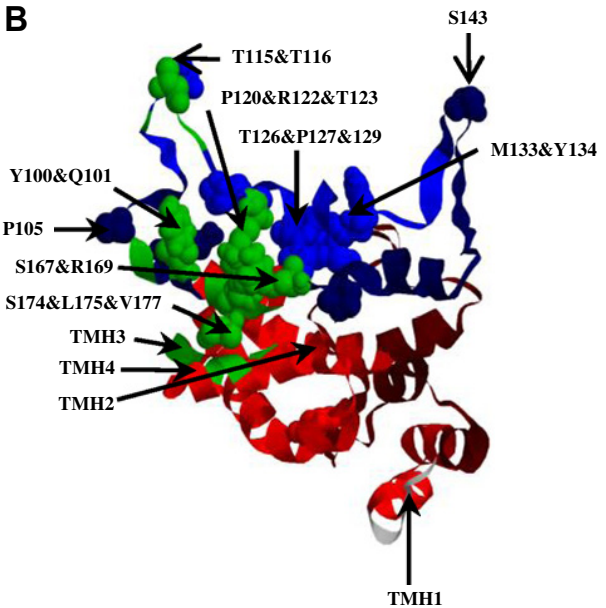

C

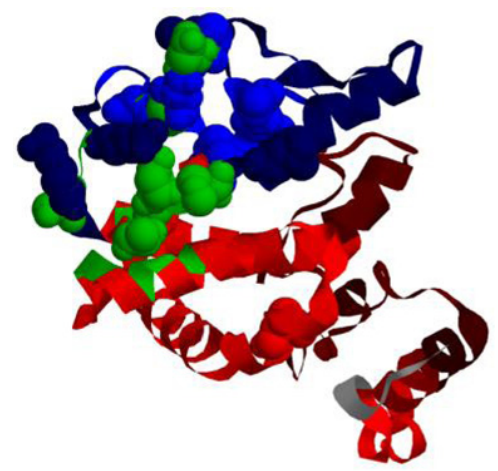

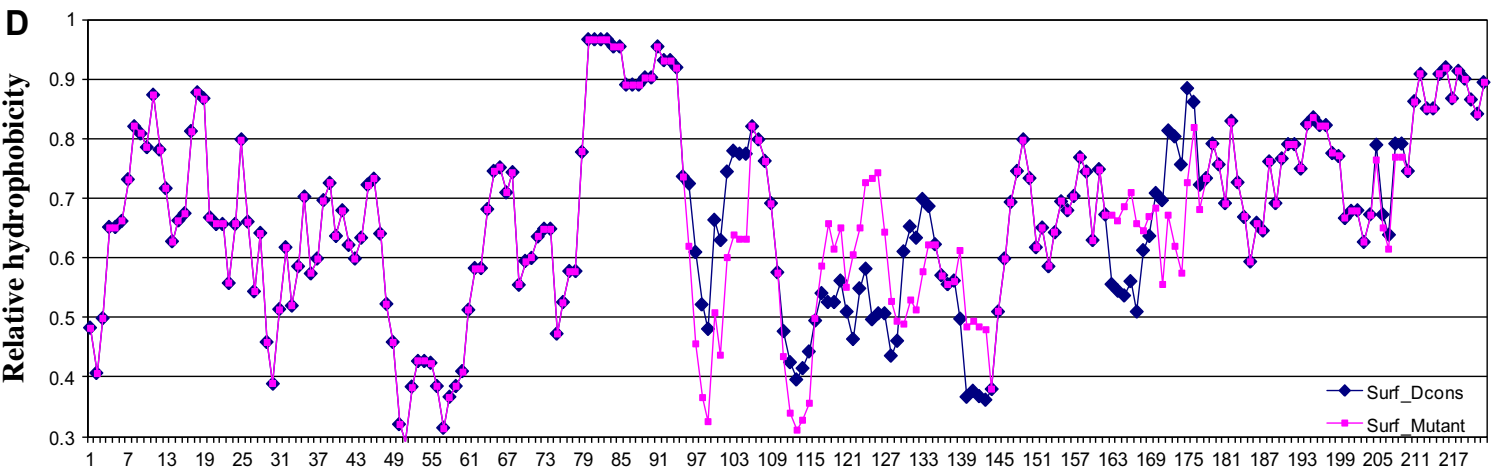

Amino acid sequence position

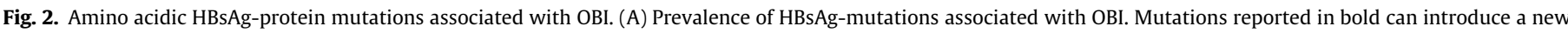

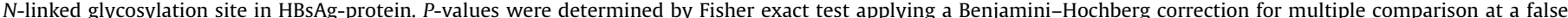

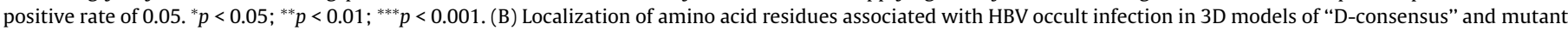

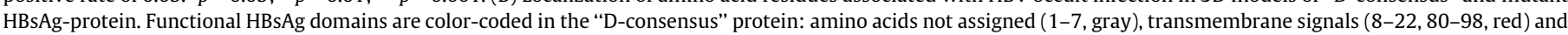

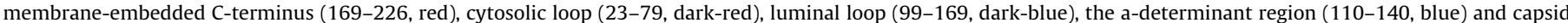

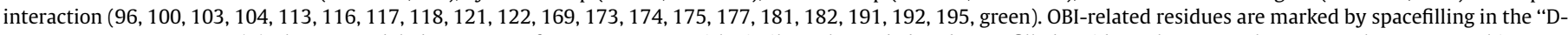

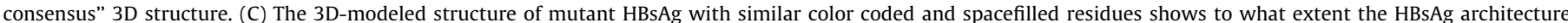

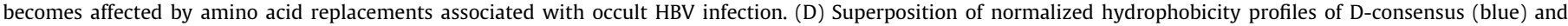

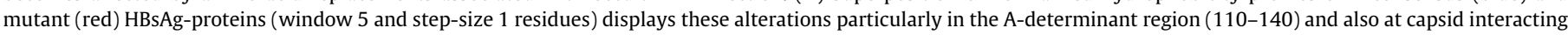
residues $(97-105,160-177)$

mutation clusters to hamper HBsAg detection was investigated in cell-culture using the ABBOTT Architect HBsAg (Qual) assay.

The detection rates of expressed HBsAg mutants significantly differed according to the pattern of mutations analyzed (Fig. 3). The supernatants of mutants Y100S and Y100S + S143L showed no detectable HBsAg ( $<1$ sample/cutoff-ratio [S/CO]). A strong decrease in the detection was also observed for HBsAg-mutants carrying T116N, T116N + S143L, R122P, R122P + Q101R, or R122P + S167L. These mutations were associated with remarkable decrease of HBsAg-reactivity from $306.8 \pm 64.1 \mathrm{~S} / \mathrm{CO}$ for wild-type to $54.9 \pm 22.6 \mathrm{~S} / \mathrm{CO}, 31.2 \pm 12.0 \mathrm{~S} / \mathrm{CO}, 6.1 \pm 2.4 \mathrm{~S} / \mathrm{CO}, 3.0 \pm 1.0 \mathrm{~S} / \mathrm{CO}$ and $3.9 \pm 1.3 \mathrm{~S} / \mathrm{CO}$, respectively ( $p<0.01$ for all tests). By contrast, 


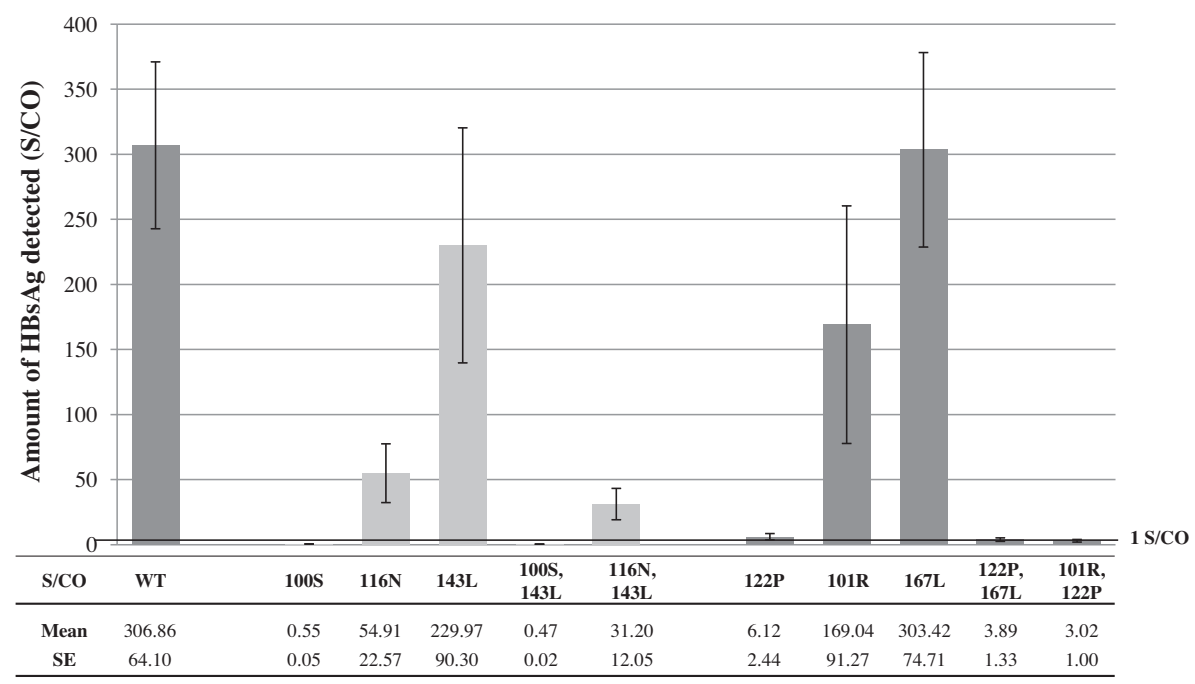

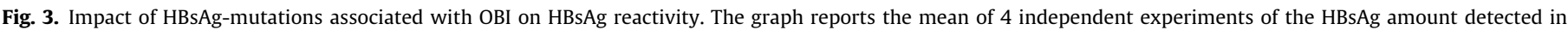

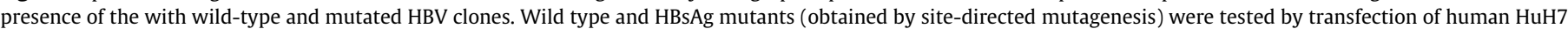

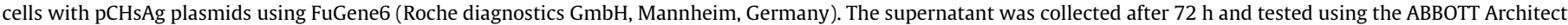

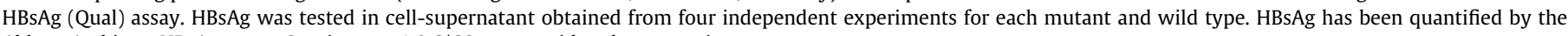
Abbott Architect HBsAg assay. Specimens $<1.0$ S/CO are considered not reactive.

HBsAg-results for Q101R, S167L and S143L were comparable to wild-type.

\section{Discussion}

This study documented a prevalence of $0.057 \%$ of HBsAg-positive HBV-DNA positive patients, and of $0.006 \%$ of $\mathrm{OBI}$ in a large cohort of blood donors tested by NAT procedure, resolved by minipools of 5/6 samples, between 2004 and 2008. This is in the range of prevalence of OBI estimated in Italy in previous studies $(0.013-$ 0.006\%) (Dettori et al., 2009; Velati et al., 2008) and in other European Countries, such as Greece $(0.0002 \%)$ and Poland $(0.084 \%)$ (Brojer et al., 2006; Katsoulidou et al., 2009).

Several possible mechanisms have been hypothesized for the pathogenesis of $\mathrm{OBI}$ and the condition is probably multi-factorial (Raimondo et al., 2008). Due to the very small group of OBIs so far analyzed, HBsAg genetic markers in D-genotype significantly associated with $\mathrm{OBI}$ in vivo are still missing.

By an integrated clinical, structural and mathematical approach, 20 mutations (18 not previously correlated, and two previously associated with HBV immune-escape, T123N and M133T) (Hou et al., 2001; Torresi, 2002) were found to be significantly associated with occult HBV D-genotype infection in vivo, mainly located in the "a-determinant" region. This is the first study showing such correlation of OBI with an HBV genotype most commonly found in western countries.

The majority of these OBI-associated HBsAg genetic-markers were completely absent in patients with chronic hepatitis. In addition, they occurred with a median number [IQR] of 3.0 [2.0-6.0] in patients with OBI. The route towards the occult state may be therefore characterized by an accumulation of variants, followed by a step-by-step loss of antigen-antibody reactivity (that is the basis of HBsAg detection test in clinical practice).

In-vitro experiments showed that specific patterns of HBsAgmutations (Y100S; Y100S + S143L; T116N + S143L; R122P; $\mathrm{R} 122 \mathrm{P}+\mathrm{Q} 101 \mathrm{R}$ and $\mathrm{R} 122 \mathrm{P}+167 \mathrm{~L}$ ) strongly decreased the amount of detected HBsAg in the supernatants, suggesting an impairment in HBsAg-detection and/or changes in the antigenicity.

The impact of such mutation-patterns on HBsAg detection has not yet been evaluated before. An important HBsAg function is the anchorage in the endoplasmatic-reticulum followed by assembly and secretion of mature virions (also HBsAg-mediated processes). Notably, transmembrane helices and the hydrophobic C-terminus are largely saved from extensive mutation in OBI. Conversely, we observed a linkage between amino acid replacement near or at positions involved in capsid interaction $(100,101,116$, $122,169,174,175,177)$, suggesting a potential ability to impair viral particle maturation and/or to hamper viral infectivity (van Hemert et al., 2008). Consistent with this hypothesis, a recent in vitro study showed the ability of mutations at position 169 (such as the $\mathrm{R} 169 \mathrm{H}$ ) to drastically hamper virion secretion, and to have a dominant negative effect when it is co-expressed with wild-type envelope proteins (Ito et al., 2010).

For mutations T115N, T116N and T123N, the correlation with OBI can be explained by the fact that these mutations introduce a novel N-liked glycosylation site, that may impair virion secretion, along with reduction of HBsAg immunogenicity; indeed T123N has been recently shown to strongly reduce virion assembly by leading to the production of a new $\mathrm{N}$-glycosylated form of $\mathrm{HBsAg}$ (Wu et al., 2010).

Residues associated with occult HBV D-genotype infection at positions $105,120,122$, and 123 have been recently shown to be critical conformation-dependent features for HBV entry into the target cells (Salisse and Sureau, 2009). Mutations at these residues have been found to drastically hamper (or even abrogate) in vitro viral infectivity (Salisse and Sureau, 2009), thus supporting their association with $\mathrm{OBI}$ in vivo.

Notably, while mutations in the "a-determinant" often resulted in non-synonymous mutations in the reverse transcriptase (RT) protein, the majority of the amino acid substitutions involving capsidinteracting residues were silent in the RT. From the evolutionary point of view, this observation points towards an accumulation of mutations due to a relaxation of selective constraints and may indicate a contribution of these regions to the evolutionary transition from a chronic into an OBI, observation also supported by previous studies (van Hemert et al., 2008) and by the higher values of evolutionary divergence and $\mathrm{dN} / \mathrm{dS}$ found in occult infection respecting to chronic. Functionally, a diminished interaction between core and surface proteins due to the mutations introduced at these regions may support this process, rendering the transition irreversible.

A recent study showed an association between OBI and immune-escape (El Chaar et al., 2005), postulating that patients with 
OBI carrying both anti-HBc and anti-HBs, are under strong humoral immune-pressure. This selecting-pressure may lead to the selection of viral strains with highly mutated $\mathrm{HBsAg}$, in particular in the HBsAg regions 125-131 and 158-169, crucial for HBsAg recognition and to reduce the exposure of HBsAg immunogenic surface (Wu et al., 2010). These mutations may be responsible for HBV evasion from the immune-system, and, at the same time, may hamper viral replication, justifying the very low serum HBV-DNA levels classically observed in OBI. Due to the quasispecies nature of HBV, the reactivation of viral replication under immune-suppression may be mainly sustained by the re-emergence of the wild-type strain.

OBI has been associated with the onset of HBV-related hepatocarcinogenesis and lymphomagenesis (Fang et al., 2009; Engels et al., 2010). Further studies are thus necessary to verify the ability of these mutations to modulate HBV oncogenic potential. In addition, recent studies have highlighted new insights regarding HBV pathogenesis (Neumann et al., 2010; Tian et al., 2010). In particular, a study shows that expression and secretion of HBsAg is a novel mode of inducing secretion of Cyclophilin A (СурA), that has been involved in the pathogenesis of HBV infection (Tian et al., 2010). Another study identifies a novel antiviral mechanism of antibodies to HBsAg involving prolonged blocking of the HBV and HBsAg subviral particles release from infected cells (Neumann et al., 2010). The impact of HBsAg mutations correlated with OBI on these two novel mechanisms underlying HBV pathogenesis should be matter of further investigation.

Specific experimental verification is warranted to provide insights on the impact of these mutations on virus maturation and replication.

A new Abbott ARCHITECT ${ }^{\circledR}$ assay for the detection of HBsAg has been recently proposed to specifically enhance the detection of some HBsAg mutants at position 122 and 123 (Lou et al., 2011). The impact of the other genetic markers we identified on the performance of this new assay has not yet been defined.

It should be noted that such study has been conducted in OBIs all carrying D-genotype HBV. Therefore, the conclusions driven by these results may not be applicable to other HBV-genotypes. Consistent with this hypothesis, a recent study, led in China, identified specific HBsAg mutations (in the regions from amino acids 117 to 121 and amino acids 144 to 147 ) potentially associated with OBIinfection in HBV B- and C-genotype. These mutations are completely different from those we identified in HBV D-genotype (none among those described in genotype $\mathrm{D}$ are reported in genotype B and C, and viceversa) (Yuan et al., 2010), thus supporting the concept that immune escape and OBI can be driven by HBVassociated mutations different for each HBV genotype. As a consequence, the natural variability of HBV genotypes may make difficult the definition of universal diagnostic markers of HBV.

In conclusions, unique HBsAg-mutations in genotype-D, different than those described in genotypes $\mathrm{B} / \mathrm{C}$ (rarely found in Western-Countries), tightly correlate with $\mathrm{OBI}$, and strongly affect HBsAg-detection. These mutations can alter HBV-antigenicity and/or viral-particle maturation, and can affect full-reliability of diagnostic-assays for HBsAg-detection. Thus, the incorporation of these mutants in reagent development can further improve the rate of true-positive results.

\section{Transparency declaration}

C.-F. Perno have received funds for attending symposia, speaking, organizing educational activities, grant research support, consultancy and advisory board membership, from Abbott, Boehringer Ingelheim, Bristol Myers Squibb, Gilead, Merck Sharp \& Dohme, Janssen Cilag, Pfizer, Tibotec, Roche, and ViiV. F. Ceccherini-Silberstein has received funds for attending symposia, speaking and organizing educational activities from Abbott, Merck Sharp \& Dohme, Janssen Cilag, and Virco. V Svicher has received funds for attending symposia, speaking and organizing educational activities from VIIV. The other authors declare that no competing interests exist.

\section{Acknowledgments}

We thank Laura Scipioni and Andrea Biddittu for data-management. This work was financially supported by grants from the Italian National Institute of Health, the Ministry of University and Scientific Research, Current and Finalized Research of the Italian Ministry of Health, and AVIRALIA Foundation. The funders had no role in study design, data collection and analysis, decision to publish, or preparation of the manuscript.

\section{Appendix A. Supplementary data}

Supplementary data associated with this article can be found, in the online version, at doi:10.1016/j.antiviral.2011.10.022.

\section{References}

Black, S.D., Mould, D.R., 1991. Development of hydrophobicity parameters to analyze proteins which bear post- or cotranslational modifications. Anal Biochem. 193, 72-82.

Blum, H.E., Galun, E., Liang, T.J., Wands, J.R., 1991. Naturally occurring missense mutation in the polymerase gene terminating hepatitis B virus replication. J. Virol. 65, 1836-1842.

Brojer, E., Grabarczyk, P., Liszewski, G., Mikulska, M., Allain, J.P., Letowska, M., 2006. Characterization of HBV DNA+/HBsAg- blood donors in Poland identified by triplex NAT. Hepatology 44, 1666-1674

Chaudhuri, V., Tayal, R., Nayak, B., Acharya, S.K., Panda, S.K., 2004. Occult hepatitis B virus infection in chronic liver disease: full-length genome and analysis of mutant surface promoter. Gastroenterology 127, 1356-1371.

Dettori, S., Candido, A., Kondili, L.A., Chionne, P., Taffon, S., Genovese, D., Iudicone, P., Miceli, M., Rapicetta, M., 2009. Identification of low HBV-DNA levels by nucleic acid amplification test (NAT) in blood donors. J. Infect. 59, 128-133.

El Chaar, Candotti, D., Crowther, R.A., Allain, J.P., 2005. Impact of hepatitis B virus surface protein mutations on the diagnosis of occult hepatitis B virus infection. Hepatology 52, 1600-1610.

Engels, E.A., Cho, E.R., Jee, S.H., 2010. Hepatitis B virus infection and risk of nonHodgkin lymphoma in South Korea: a cohort study. Lancet Oncol. 11, 827-834

Fang, Y., Shang, Q.L., Liu, J.Y., Li, D., Xu, W.Z., Teng, X., Zhao, H.W., Fu, L.J., Zhang, F.M., $\mathrm{Gu}$, H.X., 2009. Prevalence of occult hepatitis B virus infection among hepatopathy patients and healthy people in China. J. Infect. 58, 383-388.

Hoofnagle, J.H., 2009. Reactivation of hepatitis B. Hepatology 49, S156-S165.

Hou, J., Wang, Z., Cheng, J., Lin, Y., Lau, G.K., Sun, J., Zhou, F., Waters, J., Karayiannis, P., Luo, K., 2001. Prevalence of naturally occurring surface gene variants of hepatitis B virus in nonimmunized surface antigen-negative Chinese carriers. Hepatology 34, 1027-1034.

Ito, K., Qin, Y., Guarnieri, M., Garcia, T., Kwei, K., Mizokami, M., Zhang, J., Li, J. Wands, J.R., Tong, S., 2010. Impairment of hepatitis B virus virion secretion by single-amino-acid substitutions in the small envelope protein and rescue by a novel glycosylation site. J. Virol. 84, 12850-12861.

Katsoulidou, A., Paraskevis, D., Magiorkinis, E., Moschidis, Z., Haida, C., Hatzitheodorou, E., Varaklioti, A., Karafoulidou, A., Hatzitaki, M., Kavallierou, L., Mouzaki, A., Andrioti, E., Veneti, C., Kaperoni, A., Zervou, E., Politis, C. Hatzakis, A., 2009. Molecular characterization of occult hepatitis B cases in Greek blood donors. J. Med. Virol. 81, 815-825.

Levast, M., Larrat, S., Thelu, M.A., Nicod, S., Plages, A., Cheveau, A., Zarski, J.P. Seigneurin, J.M., Morand, P., Leroy, V., 2010. Prevalence and impact of occult hepatitis $B$ infection in chronic hepatitis $C$ patients treated with pegylated interferon and ribavirin. J. Med. Virol. 82, 747-754.

Lou, S.C., Pearce, S.K., Lukaszewska, T.X., Taylor, R.E., Williams, G.T., Leary, T.P., 2011 An improved Abbott ARCHITECT((R)) assay for the detection of hepatitis B virus surface antigen (HBsAg). J. Clin. Virol. 51, 59-63.

Neumann, A.U., Phillips, S., Levine, I., Ijaz, S., Dahari, H., Eren, R., Dagan, S., Naoumov, N.V., 2010. Novel mechanism of antibodies to hepatitis B virus in blocking viral particle release from cells. Hepatology 52, 875-885.

Raimondo, G., Pollicino, T., Romano, L., Zanetti, A.R., 2010. A 2010 update on occult hepatitis B infection. Pathol. Biol. (Paris) 58, 254-257.

Raimondo, G., Allain, J.P., Brunetto, M.R., Buendia, M.A., Chen, D.S., Colombo, M. Craxì, A., Donato, F., Ferrari, C., Gaeta, G.B., Gerlich, W.H., Levrero, M., Locarnini, S., Michalak, T., Mondelli, M.U., Pawlotsky, J.M., Pollicino, T., Prati, D., Puoti, M., Samuel, D., Shouval, D., Smedile, A., Squadrito, G., Trépo, C., Villa, E., Will, H., Zanetti, A.R., Zoulim, F., 2008. Statements from the Taormina expert meeting on occult hepatitis B virus infection. J. Hepatol. 49, 652-657. 
Salisse, J., Sureau, C., 2009. A function essential to viral entry underlies the hepatitis B virus "a" determinant. J. Virol. 83, 9321-9328.

Roy, A., Kucukural, A., Zhang, Y.I.-T.A.S.S.E.R., 2010. A unified platform for automated protein structure and function prediction. Nat. Protoc. 5, 725-738.

Svicher, V., Gori, C., Trignetti, M., Visca, M., Micheli, V., Bernassola, M., Salpini, R. Gubertini, G., Longo, R., Niero, F., Ceccherini-Silberstein, F., De Sanctis, G.M. Spanò, A., Cappiello, G., Perno, C.F., 2009. The profile of mutational clusters associated with lamivudine resistance can be constrained by HBV genotypes. J. Hepatol. 50, 461-470.

Tamura, K., Peterson, D., Peterson, N., Stecher, G., Nei, M., Kumar, S., 2011. MEGA5 2011: Molecular evolutionary genetics analysis using maximum likelihood, evolutionary distance, and maximum parsimony methods. Molecular Biology and Evolution.

Tian, X., Zhao, C., Zhu, H., She, W., Zhang, J., Liu, J., Li, L., Zheng, S., Wen, Y.M., Xie, Y. 2010. Hepatitis B virus (HBV) surface antigen interacts with and promotes cyclophilin a secretion: possible link to pathogenesis of HBV infection. J. Virol. $84,3373-3381$

Torresi, J., 2002. The virological and clinical significance of mutations in the overlapping envelope and polymerase genes of hepatitis B virus. J. Clin. Virol. 25, 97-106.

van Hemert, F.J., Zaaijer, H.L., Berkhout, B., Lukashov, V.V., 2008. Occult hepatitis B infection: an evolutionary scenario. Virol. J. 146 (5), 1743-1746.

Velati, C., Romano, L., Fomiatti, L., Baruffi, L., Zanetti, A.R., 2008. Impact of nucleic acid testing for hepatitis B virus, hepatitis C virus, and human immunodeficiency virus on the safety of blood supply in Italy: a 6-year survey. Transfusion 48, 2205-2213.

Vivekanandan, P., Kannangai, R., Ray, S.C., Thomas, D.L., Torbenson, M., 2008. Comprehensive genetic and epigenetic analysis of occult hepatitis B from liver tissue samples. Clin. Infect. Dis. 46, 1227-1236.

Wu, C., Zhang, X., Tian, Y., Song, J., Yang, D., Roggendorf, M., Lu, M., Chen, X., 2010 Biological significance of amino acid substitutions in hepatitis B surface antigen (HBsAg) for glycosylation, secretion, antigenicity and immunogenicity of HBsAg and hepatitis B virus replication. J. Gen. Virol. 91, 483-492.

Weinberger, K.M., Zoulek, G., Bauer, T., Bohm, S., Jilg, W., 1999. A novel deletion mutant of hepatitis B virus surface antigen. J. Med. Virol. 58, 105-110.

Yang, Z., 2007. PAML 4: phylogenetic analysis by maximum likelihood. Mol. Biol. Evol. 24, 1586-1591.

Yuan, Q., Ou, S.H., Chen, C.R., Ge, S.X., Pei, B., Chen, Q.R., Yan, Q., Lin, Y.C., Ni, H.Y., Huang, C.H., Yeo, A.E., Shih, J.W., Zhang, J., Xia, N.S., 2010. Molecular characteristics of occult hepatitis B virus from blood donors in southeast China. J. Clin. Microbiol. 48, 357-362.

Zaaijer, H.L., Torres, P., Ontañón, A., Ponte, L.G., Koppelman, M.H., Lelie, P.N., van Hemert, F.J., Boot, H.J., 2008. Multiple surface antigen mutations in five blood donors with occult hepatitis B virus infection. J. Med. Virol. 80, 1344-1349.

Zhang, J., Hou, T., Wang, W., Liu, J.S., 2010. Detecting and understanding combinatorial mutation patterns responsible for HIV drug resistance. Proc. Natl. Acad. Sci. USA 107, 1321-1326. 